\title{
Suppression of Tunneling into Multiwall Carbon Nanotubes
}

\author{
A. Bachtold,* M. de Jonge, K. Grove-Rasmussen, and P. L. McEuen \\ Department of Physics, University of California at Berkeley, Berkeley, California 94720 \\ and Materials Sciences Division, Lawrence Berkeley National Laboratory, Berkeley, California 94720
}

M. Buitelaar and C. Schönenberger

Institut für Physik, Universität Basel, Klingelbergstrasse 82, CH-4056 Basel, Switzerland

(Received 5 December 2000; published 1 October 2001)

\begin{abstract}
We have studied tunneling of electrons into multiwall carbon nanotubes (NTs) in NT-gold and NT-NT junctions, the latter created by atomic force microscope manipulation. The tunneling conductance goes to zero as the energy (temperature and bias) is reduced, and the functional form is consistent with a power law. The exponents depend upon sample geometry. The relationship between these results and theories for tunneling into ballistic and disordered metals is discussed.
\end{abstract}

DOI: $10.1103 /$ PhysRevLett.87.166801

Carbon nanotubes (NTs) are emerging as an excellent system for the investigation of electronic transport in one dimension (1D). Two different classes of NTs exist: small diameter $(\approx 1 \mathrm{~nm})$ single-wall NTs (SWNTs) and large diameter $(\approx 10 \mathrm{~nm})$ multiwall NTs $($ MWNTs). Metallic SWNTs are characterized by two $1 \mathrm{D}$ channels and long mean free paths: $l>1 \mu \mathrm{m}$ [1-3]. As such, they represent a nearly perfect $1 \mathrm{D}$ system. It is well known that in 1D transport is strongly affected by electron-electron $(e-e)$ interaction, producing a system called a Luttinger liquid (LL) whose low energy states are collective in nature $[4,5]$. The tunneling density of states (tunneling DOS $=$ TDOS) of a LL diminishes as a power law with energy according to $\nu \propto E^{\alpha}$ with different exponents $\alpha$ for different geometries, e.g., whether the electron tunnels into the end or the bulk. The tunneling conductance $G \propto$ TDOS has been measured for SWNTs in a variety of geometries and good agreement with LL predictions has been achieved [6-8].

Various experiments on the transport properties of MWNTs have been performed. Experiments with low resistance contacts show interference effects, such as Aharonov-Bohm oscillations $[9,10]$, weak localization, and universal conductance fluctuations [10,11]. These have been used to infer the mean free path, yielding $l \lesssim$ $100 \mathrm{~nm}$. They also indicate that the dc current is predominantly carried in the outer shell of the NT. Similarly, transport $[10]$ and scanned probe experiments $[3,12]$ indicate a typical resistance per unit length of $5-10 \mathrm{k} \Omega / \mu \mathrm{m}$.

In this Letter we discuss measurements of the tunneling conductance of MWNTs. In one geometry, metallic contacts to the tube with high resistance are employed. In a second geometry, NT-NT junctions are created by manipulation with an atomic force microscope (AFM). The tunneling conductance is measured as a function of temperature and bias voltage. These measurements show that also for MWNTs the TDOS is a power law over a wide energy range of $1 \lesssim E \lesssim 100 \mathrm{meV}$ with geometrydependent exponents, which surprisingly agree quite well with those for SWNTs. This suggests a similar origin.
PACS numbers: 73.50.-h, 72.15.Nj, 73.61.Wp

Unlike SWNTs, however, MWNTs are diffusive or at best quasiballistic, so that the applicability of LL theory must be carefully considered.

Power laws in tunneling may also be caused by the disorder-enhanced Coulomb interaction, as was treated perturbatively in the seminal work of Altshuler, Aronov, and Lee (AAL) [13]. Importantly, perturbation theory does not suffice for MWNTs since the observed corrections are large. A nonperturbative treatment, applied to the specific geometry of MWNTs, has recently been put forward by Egger and Gogolin (EG) [14]. Similar to LL theory, a power law is predicted. We therefore compare our measurement with both the EG and the LL model.

The MWNTs were synthesized by arc-discharge evaporation and deposited from a dispersion in chloroform onto an oxidized Si wafer. NTs with diameters ranging from 8 to $17 \mathrm{~nm}$ are selected and located using a scanning electron microscope or an AFM. For devices of the first type, gold contacts to the tube are then created using $e$-beam lithography. This procedure typically leads to contacts with low resistance $(\approx 1 \mathrm{k} \Omega)$, but it also occasionally produces highly resistive contacts $(>10 \mathrm{k} \Omega)$. The microscopic origin of the high resistance is not known. Here, however, we merely exploit these accidental tunnel barriers to probe the electronic properties of the MWNT.

The inset in Fig. 1 shows a schematic of a device consisting of a $d=17 \mathrm{~nm}$ diameter MWNT contacted to three electrodes. The $4.5 \mathrm{k} \Omega$ resistance measured between the two outer electrodes corresponds to the typical intrinsic resistance for a MWNT contacted with electrodes that are separated by $700 \mathrm{~nm}$. This indicates that the contact resistances of the two outer electrodes are low and that the NT connecting them is electrically continuous. However, the resistance measured from the inner electrode to either of the outer electrodes is much higher, $140 \mathrm{k} \Omega$. This inner electrode has thus low transparency and serves as a tunneling contact to explore the TDOS of the tube.

Figure 1 shows a series of measurements of the tunneling conductance versus bias for different magnetic fields 


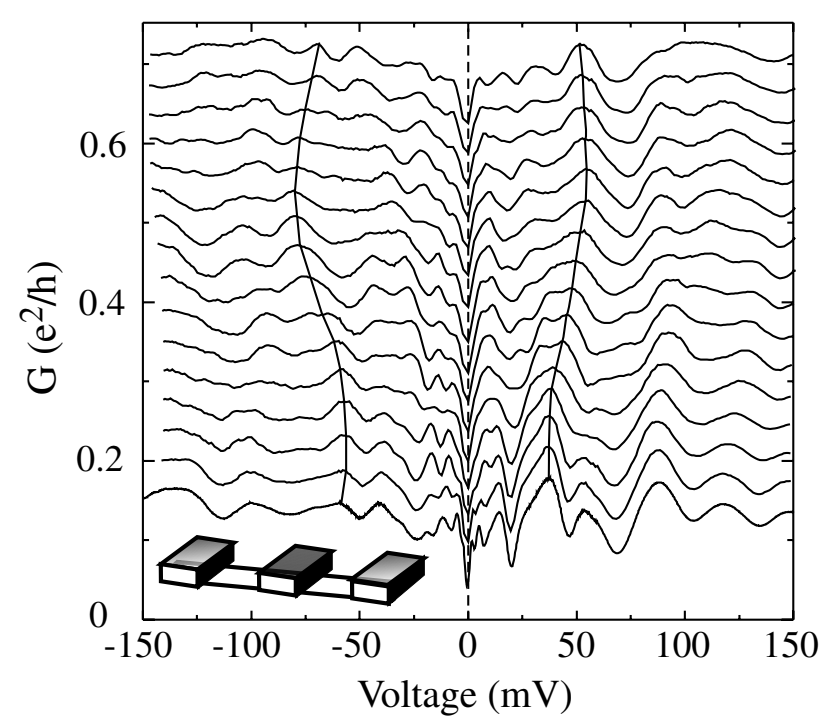

FIG. 1. $G=d I / d V$ as a function of $V$ at $2 \mathrm{~K}$ for different parallel magnetic fields $B=0,1, \ldots, 15 \mathrm{~T}$. Curves are offset for clarity. The movement with $B$ of two well discernible peaks is indicated by solid curves. Inset: schematic of MWNT-Au devices with three electrodes, here separated by $350 \mathrm{~nm}$. The inner electrode has a high resistance and serves as tunneling contact for measuring $d I / d V$.

$B$ applied parallel to the tube. The $d I / d V$ spectra are highly structured. We first note that, at small energies $(0-10 \mathrm{mV})$ the spectra display a strongly suppressed conductance. This suppression is centered at $V=0$ independent of $B$. Before discussing this anomaly, we first briefly address the complex features at higher energy. These features are quasiperiodic, with an average spacing between the maxima of $\approx 25 \mathrm{mV}$ and they evolve with increasing magnetic field.

The observed features are likely a consequence of quasione-dimensionality. One-dimensional states (so called 1D subbands) have a pronounced energy-dependent DOS with peaks at the subband threshold (Van Hove singularity) [15]. Here, the peaks are broadened due to scattering from disorder. This assignment is supported by the observed peak spacing of $\approx 25 \mathrm{mV}$ which is in good agreement with the expected spacing of $\hbar v_{F} / d=29 \mathrm{meV}\left(v_{F}=\right.$ $8 \times 10^{5} \mathrm{~m} / \mathrm{s}$ is the Fermi velocity, $d=17 \mathrm{~nm}$ ). The identification of these peaks as broadened Van Hove peaks is further supported by their behavior in $B$. With evolving parallel field $B$ the position of the peaks move in energy up and down, as expected from the Aharonov-Bohm effect [16]. Theory predicts a periodic movement with a fundamental period $h / e$ which translates into a field of $\approx 17 \mathrm{~T}$. Though the level moves on the expected field scale, the peak movement is not simple in Fig. 1. The reason is not clear yet, but it may be attributed to disorder and inhomogeneities. The emergence of broadened Van Hove peaks in the TDOS demonstrates that the elastic length $l$ is of the same order as the circumference of the tube. The MWNTs are therefore not in the two-dimensional diffusive regime $(l<d)$.
We now turn to the low bias suppression of the conductance. It is independent of $B$, which suggests a different origin than the peaks discussed above. In addition, its dependence on $V$ and $T$ is very different, as we now discuss. Figure 2 shows the tunneling conductance $G=d I / d V$ of a second, similarly prepared MWNT sample. A conductance dip centered at zero bias (zero-bias anomaly $=\mathrm{ZBA}$ ) is again observed in $G(V)$ (Fig. 2a). As $T$ is decreased down to $350 \mathrm{mK}$, the amplitude of the dip increases. In Fig. $2 b$ the zero-bias conductance is plotted as a function of $T$ in a double logarithmic plot, demonstrating that the measured data can be well described by a power law $G \propto T^{\alpha}$ with exponent $\alpha=0.36$. For bias voltages larger than $k_{B} T / e$, the voltage dependence can also be described by a power law with the same exponent 0.36 . This can be seen in Fig. 2c, which shows a double-logarithmic plot of the symmetrical part of $G$ divided by $T^{\alpha}$ as a function of $e V / k_{B} T$. All data collapse on a single universal curve, similar to what has been observed in SWNTs [6]. Power-law scaling in $T$ and $V$ has been found in 11 different samples with exponents $\alpha$ ranging from 0.24 to 0.37 .

To explore this ZBA further, we created devices composed of two MWNTs arranged in different geometries. An AFM tip has been used to move NTs $[8,17]$. The end of one tube is pushed against either the end or the middle (bulk) of a second tube. Au contacts are attached to both tubes. Examples of end-bulk and end-end junctions are shown in Figs. 3a and 3b, respectively. The resistance values of these junctions vary considerably, from immeasurably large to $\approx 100 \mathrm{k} \Omega$. These large values suggest that the junction between two tubes serves as a tunnel barrier.

As in metal-NT junctions, pronounced ZBAs are present in all junctions. However, the suppression is significantly more dramatic in NT-NT as compared to Au-NT junctions. Figure 4a shows $d I / d V$ as a function of $V$ in a double

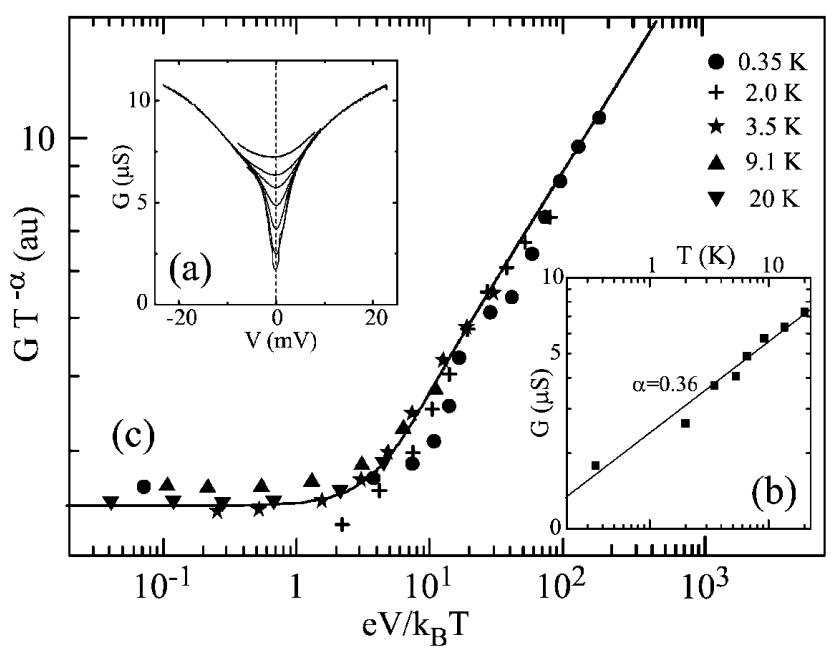

FIG. 2. (a) $G(V, T=$ const $)=d I / d V$ of a second MWNT for $T=0.35, \ldots, 20 \mathrm{~K}$. (b) The linear conductance $G(0, T)$ in a double logarithmic plot demonstrating power-law scaling. (c) $G(V, T) T^{-\alpha}$ versus $e V / k_{B} T$. Similar to the $T$ dependence, $G \propto V^{\alpha}$ for $e V \gg k_{B} T$ with power $\alpha=0.36$. 


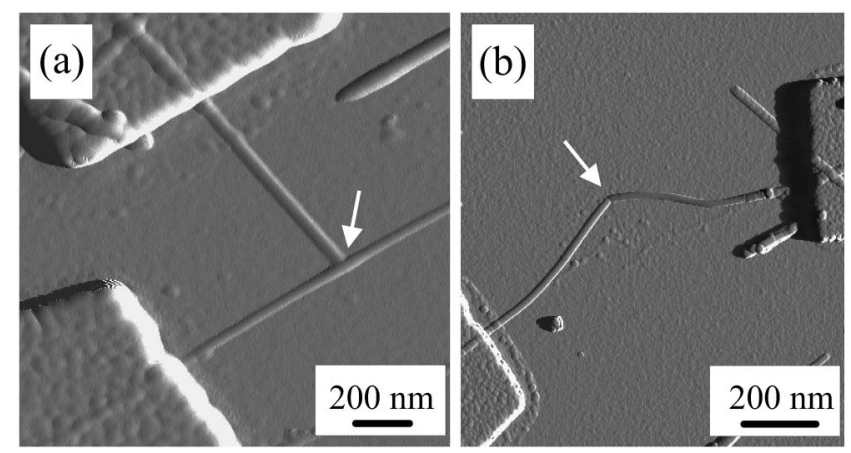

FIG. 3. AFM images of junctions formed between two MWNTs: (a) end-bulk junction and (b) end-end junction. The arrows indicate the position of the junctions.

logarithmic scale at $T=3 \mathrm{~K}$ for a bulk-end and an end-end junction. For comparison, the tunneling conductance of a typical Au-NT junction is also plotted. The curves show approximate power-law behavior, but with different exponents. We find $\alpha=0.9$ and 1.24 for bulk-end and end-end NT-NT junctions. These exponents are representative of seven junctions studied.

Overall, our result for tunneling into MWNTs can be summarized by a simple rule. The conductance is given by $G \sim E^{\alpha}$, where $E$ is the excess energy of the tunneling electron, given by the larger of $e V$ or $k_{B} T$. The exponent $\alpha$ can be approximated by $\alpha=\alpha_{1}+\alpha_{2}$, where $\alpha_{1,2}$ represent the properties of the conductor on either side of the junction: $\alpha^{\text {bulk }} \sim 0.3$ for the tube bulk, $\alpha^{\text {end }} \sim 2 \alpha^{\text {bulk }} \sim 0.6$ for the tube end, and $\alpha \sim 0$ for the Au contact, respectively.

We now discuss the possible origins of the ZBA. The voltage and temperature dependence of $G$ could be caused by the energy dependence of the single particle DOS of graphene, the 2D material from which NTs are made. The 2D DOS of graphene is $\propto E$, which would relate into $\alpha^{\text {bulk }}=1$, in contradiction to the observation. Moreover, if the observed anomaly would be related to the single
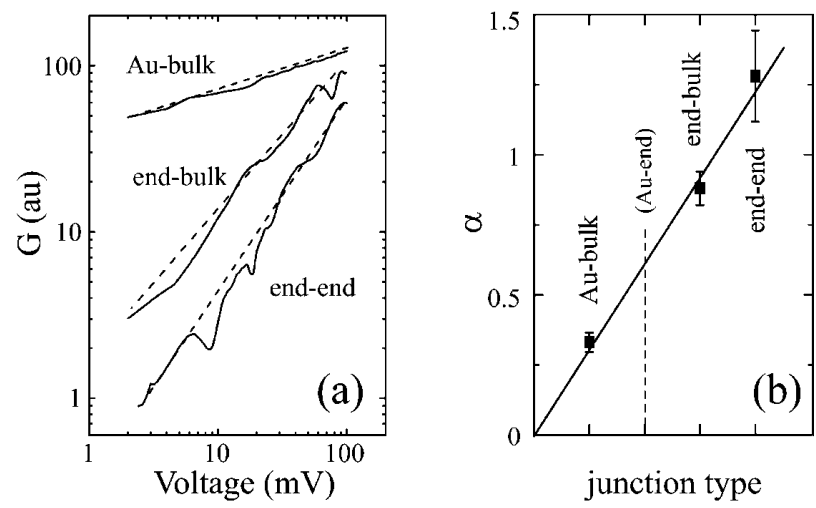

FIG. 4. (a) $G$ as a function of $V$ in a double logarithmic plot for a Au-bulk, an end-bulk, and an end-end junction. The corresponding slopes are $\alpha=0.25,0.9$, and 1.24, respectively. (b) Plot of $\alpha$ as a function of the junction type for $\mathrm{Au}-\mathrm{Au}$, Au-bulk, Au-end, bulk-end, and end-end junctions. particle DOS, its position would depend on gate voltage (back gate), which is not observed.

A ZBA in the TDOS is often taken to be a signature of $e$ - $e$ interactions. For example, the correction (suppression) to the density of states $\nu$ due to $e-e$ interaction has been calculated by AAL perturbatively, valid if $\delta \nu \ll \nu$ or $\delta G \ll G$ [13]. This interpretation has been successful in describing ZBAs in tunnel contacts to disordered metals [18]. In contrast to these earlier experiments, we report on anomalies with large amplitudes $\delta G \sim G$. A quantitative understanding requires to calculate the TDOS nonperturbatively in the interaction.

Nonperturbative treatments exist for a number of cases. In the case of a clean 1D quantum wire, the $e$-e interaction can be accounted for analytically at any strength leading to a Luttinger liquid [4]. The case of a tunnel junction whose coupling to the ideal reservoirs is described by a frequency dependent impedance $Z(\omega)$ has also been studied extensively [19]; we refer to this as the environmental Coulomb blockade theory (ECBT). The LL and ECBT theories bear a lot in common. Tunneling into a LL excites plasmon modes which can be understood as the eigenmodes of an $L C$-transmission line, where $L$ is the kinetic inductance and $C$ is the external electrostatic capacitance. The impedance $Z$ of such an ideal transmission line is Ohmic with $Z=R=\sqrt{L^{\prime} / C^{\prime}}$, where $L^{\prime}$ and $C^{\prime}$ denote inductance and capacitance per unit length. In the limit of many modes in parallel, both theories give $G \propto V^{\alpha}$ (at $T=0$ ) with $\alpha^{\text {end }}=2 R / R_{K}$, where $R_{K}=h / e^{2}$ is the quantum resistance [20,21].

These theories also predict $\alpha^{\text {end }} / \alpha^{\text {bulk }}=2$ for a large number of modes. The factor 2 reflects the fact that a quasiparticle added to the bulk can propagate away into the "right" and "left" arm of the NT, while it can propagate only in one arm if added to the end. In the spirit of ECBT the effective environmental impedance is halved. This can be generalized to other geometries. In terms of $\alpha^{\text {bulk }}$, both theories predict $\alpha^{\text {end }}=2 \alpha^{\text {bulk }}, \alpha^{\text {bulk-end }}=3 \alpha^{\text {bulk }}$, and $\alpha^{\text {end-end }}=4 \alpha^{\text {bulk }}$.

The limit of many modes applies to our experiment, since MWNTs have recently been shown to be considerably hole doped [22]. Because of doping $N \approx 10-20$ subbands are occupied, instead of four for an ideal SWNT. The large- $N$ LL/EBCT theory should therefore apply. Indeed the data are in very good agreement with the predictions. The power-law exponents increase as predicted with changes in geometry; see Fig. $4 \mathrm{~b}$ (solid line). We can also numerically estimate the exponent. The kinetic inductance is given by $L^{\prime}=R_{K} / 2 N v_{F}$, where $v_{F} \approx 10^{6} \mathrm{~m} / \mathrm{s}$ is the Fermi velocity and $N \approx 10-20$ the number of modes, yielding $L^{\prime} \approx 1 \mathrm{nH} / \mu \mathrm{m}$. A typical value for the (external) capacitance of a nanotube is $C^{\prime}=30 \mathrm{aF} / \mu \mathrm{m}$. These values yield a transmission line impedance of $R \approx 5.7 \mathrm{k} \Omega$ and consequently a bulk exponent of $\alpha^{\text {bulk }} \approx 0.22$, a value which is in reasonable agreement with the measured exponents. It therefore appears that the ECBT assuming a fixed-impedance environment (such as an $L C$ transmission 
line), or equally, the LL theory in the large- $N$ limit, explains our observations.

So far, however, we ignored that the MWNTs are disordered conductors. Because MWNTs are disordered, it is more appropriate to model the NT as an $R C L$ transmission line rather than an $L C$ one. Let us estimate the significance of the resistance. We compare $\omega L^{\prime}$ with $R^{\prime} \approx 5 \mathrm{k} \Omega / \mu \mathrm{m}$. The inductive part is equal to the resistive part for $\hbar \omega=3.0 \mathrm{meV}$. Since the power law is observed for larger voltages, the resistive part can in fact be neglected. Therefore, the $L C$ transmission line model is applicable for not too small voltages.

We now compare our data with a nonperturbative treatment of the disorder-enhanced renormalization of the TDOS caused by intra-Coulomb interaction. This topic has only recently become the subject of intensive theoretical studies [14,23,24]. For MWNTs this problem has been solved by Egger and Gogolin [14]. They predict a power law with exponent $\alpha^{\text {end }}=$ $2 \alpha^{\text {bulk }}=\left(r / \pi D \hbar \nu_{0}\right) \log \left(1+\nu_{0} U_{0}\right)$, where $U_{0}$ is the 1D intra-Coulomb interaction, $r$ is the radius of the tube, and $D$ is the diffusion constant. The $\log ()$ term turns out to be $\sim 2-3$ and the (bare) DOS is $\nu_{0}=$ $N^{\star} / 2 \pi \hbar v_{F}$. Here, $N^{\star}$ denotes the number of modes not counting the spin and $K-K^{\prime}$ degeneracy of the graphene lattice, i.e., $N^{\star}=N / 4 \approx 5$. This yields $\alpha^{\text {bulk }} \approx$ $5 r / N^{\star} l_{e}=20 r / N l_{e}$. This equation can be expressed solely in terms of measured quantities by noting that $R^{\prime}=R_{K} / N l_{e}, \quad$ yielding $\alpha^{\text {bulk }} \approx 20 r R^{\prime} / R_{K}=$ $0.02, \ldots, 0.08$. The typical value is roughly a factor of 5 smaller than observed in the experiment. As a result, the large- $N$ LL/EBCT model is the more likely cause of the observed suppression of tunneling.

In conclusion, the tunneling DOS of MWNTs is renormalized by $e$-e interaction leading to a suppression of the tunneling conductance for small energies with power-law scaling. The observed power laws can be explained by environmental Coulomb blockade theory in which the tube acts as an effective $L C$-transmission line in series with the tunnel junction. The observed power laws are qualitatively also captured by a recent nonperturbative treatment of disorder-enhanced corrections to the DOS due to intra-Coulomb interaction. The comparison of the measured exponents with theoretical predictions favors the $L C$ transmission line model. Further experimental work is needed to separate these two contributions.

We acknowledge D. Averin, R. Egger, M. Fuhrer, E. Graugnard, P. Kim, Y. Nazarov, M. Paalanen, and S. Tans for discussions. We thank L. Forró, A. Rinzler, J.-P. Salvetat, and R. Smalley for the NT materials. This work was supported by DOE, by DARPA, and by the Swiss NSF.

*Present address: TUDelft, 2628CJ Delft, The Netherlands. [1] C. T. White and T. N. Todorov, Nature (London) 393, 240 (1998).
[2] S. J. Tans, M. H. Devoret, H. Dai, A. Thess, R. E. Smalley, L. J. Geerligs, and C. Dekker, Nature (London) 386, 474 (1997); M. Bockrath, D. H. Cobden, P. L. McEuen, N. G. Chopra, A. Zettl, A. Thess, and R. E. Smalley, Science 275, 1922 (1997).

[3] A. Bachtold, M. S. Fuhrer, S. Plyasunov, M. Forero, E. H. Anderson, A. Zettl, and P. L. McEuen, Phys. Rev. Lett. 84, 6082 (2000).

[4] M. P. A. Fisher and L. I. Glazman, in Mesoscopic Electron Transport, edited by L.L. Sohn, L. Kouwenhoven, and G. Schön (Kluwer Academic Publishers, Dordrecht, 1997); J. Voit, Rep. Prog. Phys. 57, 977 (1995).

[5] R. Egger and A. O. Gogolin, Phys. Rev. Lett. 79, 5082 (1997); C. Kane, L. Balents, and M. P. A. Fisher, Phys. Rev. Lett. 79, 5086 (1997).

[6] M. Bockrath, D. H. Cobden, Lu Jia, A. G. Rinzler, R.E. Smalley, L. Balents, and P.L. McEuen, Nature (London) 397, 598 (1999).

[7] Z. Yao, H. W. C. Postma, L. Balents, and C. Dekker, Nature (London) 402, 273 (1999).

[8] H. W. C. Postma, M. de-Jonge, and C. Dekker, Phys. Rev. B 62, R10653 (2000).

[9] A. Bachtold, C. Strunk, J.-P. Salvetat, J. M. Bonard, L. Forró, T. Nussbaumer, and C. Schönenberger, Nature (London) 397, 673 (1999).

[10] C. Schönenberger, A. Bachtold, C. Strunk, J.-P. Salvetat, and L. Forró, Appl. Phys. A 69, 283 (1999).

[11] L. Langer, V. Bayot, E. Grivei, J.-P. Issi, J. P. Heremans, C. H. Olk, L. Stockman, C. Van-Haesendonck, and Y. Bruynseraede, Phys. Rev. Lett. 76, 479 (1996).

[12] H. Dai, E. W. Wong, and C. M. Lieber, Science 272, 523 (1996).

[13] B. L. Altshuler and A. G. Aronov, Solid State Commun. 36, 115 (1979); B. L. Altshuler, A. G. Aronov, and P. A. Lee, Phys. Rev. Lett. 44, 1288 (1980).

[14] R. Egger and A. O. Gogolin, Phys. Rev. Lett. 87, 066401 (2001).

[15] J. W. G. Wildöer, L. C. Venema, A. G. Rinzler, R. E. Smalley, and C. Dekker, Nature (London) 391, 59 (1999); T. W. Odom, Jin-Lin-Huang, P. Kim, and C. M. Lieber, Nature (London) 391, 62 (1999).

[16] H. Ajiki and T. Ando, J. Phys. Soc. Jpn. 62, 1255 (1993).

[17] T. Hertel, R. Martel, and P. Avouris, J. Phys. Chem. B 102, 910 (1998); L. Roschier, J. Penttila, M. Martin, P. Hakonen, M. Paalanen, U. Tapper, E. I. Kauppinen, C. Journet, and P. Bernier, Appl. Phys. Lett. 75, 728 (1999).

[18] Y. Imry and Z. Ovadyahu, Phys. Rev. Lett. 49, 841 (1982).

[19] M. H. Devoret, D. Esteve, H. Grabert, G.-L. Ingold, H. Pothier, and C. Urbina, Phys. Rev. Lett. 64, 1824 (1990); S. M. Grivin, L. I. Glazman, M. Johnson, D. R. Penn, and M. D. Stiles, Phys. Rev. Lett. 64, 3183 (1990).

[20] K. A. Mateev and L. I. Glazman, Phys. Rev. Lett. 70, 990 (1993).

[21] R. Egger, Phys. Rev. Lett. 83, 5547 (1999).

[22] M. Krüger, M. R. Buitelaar, T. Nussbaumer, C. Schönenberger, and L. Forró, Appl. Phys. Lett. 78, 1291 (2001).

[23] A. Kamenev and A. Andreev, Phys. Rev. B 60, 2218 (1999).

[24] J. Rollbühler and H. Grabert, Phys. Rev. Lett. 87, 126804 (2001); E. G. Mishchenko, A. V. Andreev, and L. I. Glazman, cond-mat/0106448. 